\title{
Psychiatry in Medical Education
}

The concentration of medicine during the past 75 years on the conquest of infectious diseases, on the science of bacteriology, and on the contributions of physics and chemistry to biology has tended toward a medical view of man as an isolated biological unit.

A somewhat similar isolationism accompanied the recent accelerated growth of psychiatric knowledge. In the efforts to establish diagnostic techniques, there was a tendency to equate the patient with the symptoms and patterns of behavior accompanying his illness. Concentration on the work of defining, limiting, and clarifying meant that, for a time, psychiatry, too, developed as a compartmentalized medical discipline and lost sight of the simple fact that the patient is a unique human being, a person subject to a variety of physical and emotional ills.

Following the tradition of Adolph Meyer and William Alanson White, forward-looking psychiatrists have long been pointing to the need to return to the main stream of medicine and reintegrate knowledge about psychic and somatic factors. They recognize that few patients fit into the classical clinical patterns of mental illness and that, to treat the patient, all factors affecting the personality of the individual must be considered. In this, physical complaints are as important as emotional factors.

Dissatisfaction with the fragmented view of the patient has grown with the realization that, to be effective, medical treatment must do more than concentrate on the disease entity. The whole person must be treated. Consideration must be given to factors of personality and behavior which influence and are influenced by physiological condition; consideration must also be given to the environment of the person under treatment. The effect of interpersonal relations and cultural forces on the individual must not be overlooked.
The medical profession is, in a sense, "rediscovering" what historically has always been the goal of medicine-to treat the person as well as the disease. Psychiatry, as a branch of medicine, is serving as the catalyst in bringing about the changes required by this "rediscovery," for "nothing that is human is foreign to psychiatry." Its particular focus gives psychiatry a strategic role in the movement of medicine toward dealing with the patient as a person.

\section{The Practicing Physician}

The conference reemphasized the social responsibilities of the physician. It was agreed that the community has a right to expect the physician to perform his functions with an understanding of the individual patient's emotional and social problems-that the community has a right to expect preventive medicine.

The physician has obligations to his patient's family and his patient's environment as well as to his patient. Problems related to child growth, the aged, chronic illness, and problems related to the supply of physicians require thought and action by both the community and the profession of medicine. The physician must be aware of the contributions of professions other than that of medicine. He must work with members of those professions if his own contributions are to be most effective.

A major purpose of psychiatric teaching is to prepare the physician for his most effective contribution to the community by providing a cross-fertilization between medical science and social science with emphasis on the importance of understanding people as human beings. The concept of the physician and the patient as total persons in a total environment involves a humanistic even more than a medical approach. 
The growing trend in modern medicine toward a reemphasis of the need to treat the patient as a whole person was pointed up sharply in the Conference on Psychiatric Education held at Cornell University, Ithaca, N. Y., in June 1951.

Organized and conducted by the American Psychiatric Association and the Association of American Medical Colleges under a grant from the National Institute of Mental Health of the Public Health Service, the conference formally stated its purpose as the promotion and preservation of the health of the community "by investigating, defining, and helping to develop programs which will improve the teaching of basic and clinical psychiatry; by advancing the medical skills needed to recognize and treat mental illness and emotional maladjustment; by coordinating the efforts of all groups concerned with these problems so that their total resources may be used most effectively."

The recurrent theme throughout the conference, as contained in its report published in June 1952 by the American Psychiatric Association under the title of "Psychiatry and Medical Education," was the role of psychiatry in the development of integrated medical teaching. The main lines of discussion, with emphasis on public health implications, are summarized here on the basis of the published report.

Dr. Seymour D. Vestermark, author of the summary, is chief of the training and standards branch of the National Institute of Mental Health and was a participant in the conference.

\section{Infegrated Medical Education}

It was suggested in conference discussions that psychiatry could lead in coordinating medical teaching in some areas. One area is that of the physician-patient relationship, basic to all of medicine. The department of psychiatry should be responsible for this teaching area because of its special insight into the dynamics of interpersonal relations. But special attention would need to be given to integrating the department of psychiatry with the other departments in the medical center.

Similarly, another area in which psychiatry can be helpful-interviewing and history taking-presents problems which stress the need for integrated administration and crossfertilization among the different departments of the medical school. Case histories written by psychiatric residents tend to lack critical medical information, whereas case histories written by medical residents tend to bypass emotional attitudes and unconscious factors. It was suggested that the kind of interdisciplinary program needed to teach interviewing and history taking should be supported and developed by frequent communication among faculty members and between students and faculty.

\section{Research Opportunities}

For psychiatry, one of the broader implications of a rounded medical education is the continuing need and opportunity for research to validate the empirical body of knowledge acquired through clinical practice. The conference recognized the value, for medical instruction in psychiatry, of the body of knowledge derived from intensive treatment of patients whose symptoms are classifiable. But to prevent teaching from becoming mere indoctrination, it is essential that clinical knowledge be supplemented by related research, such as animal studies, investigations into the physiological and biochemical components of emotional health and emotional disturbances, and controlled experiments on the role of the learning process in psychotherapy.

On the other hand, the emphasis of psychi$\operatorname{atr} y$ on the social aspects of medicine has farreaching implications for a redirected program of medical training. Current thinking that emotional maladjustment has its roots and first manifests itself in early childhood points to the need for integrating the study of pediatrics and psychiatry, and for including the subject of growth and development in undergraduate medical instruction in psychiatry. Many medical schools are experimenting with courses in growth and development taught jointly by the departments of pediatrics and psychiatry. This is but one example of how the complex nature of social influences on physical health and disease might better be taken into account. 
Psychiatry thus opens the door for contributions from the social sciences.

\section{Human Ecology and Human Personality}

A significant portion of the conference was devoted to discussing the need for including study of human ecology and human personality in the medical curriculum. Predicated on the assumption that to fully understand an organism it is essential to understand the organism's environment, the study of human ecology would include material from social anthropology, psychology, and sociology. This material, together with material drawn directly from the field of psychiatry on human personality and on human ecology, would complement the study of human biology in the medical training program.

In listing some of the more important components of the study of human ecology and personality, the report of the conference groups topics under four major headings. Those areas of personality closely related to the study of physiology include perception, learning, emotion and motivation, and language and thought. The study of normal child behavior, and of adolescence, maturity, and senescence constitute the segment of human ecology concerned with the genesis and decline of complex human activities. The third grouping - the nature and development of personality, and the study of individual differences-is followed by a fourth grouping devoted to society and culture-the family, the interaction of personality and culture, and the forces contributing to social organization and disorganization.

\section{Coordination}

The task of incorporating such broad areas of knowledge into the medical curriculum presents some major problems. While instruction must be focused on the primary goal of producing adequately prepared physicians, it must also be designed to fit all the related parts of medicine into a coordinated presentation. A special group will need to be organized whose function it will be to insure effective teaching of human ecology and personality. This means adding to the faculty physicians with training in the social sciences and social scientists who have had experience in a clinical setting. Together, these teams might be able to cooperate on research projects and join forces in coordinating medical teaching.

The conference report indicates that currently there is a growing tendency toward integrative teaching in medical centers-sometimes involving cooperation with departments in other schools of the university. Most schools also are attempting to include instruction in psychiatry in all 4 years of the medical curriculum. Since psychiatry is linked with the practice of medicine generally and, in particular, with such areas of medical practice as pediatrics, internal medicine, endocrinology, and neurology, and since it must take into account social, cultural, and environmental considerations, it can serve as an excellent means of integrating the fragmented approach of medicine and of bringing medicine closer to the social sciences and related disciplines.

In considering techniques that might be used for integrating instruction, the conference placed much emphasis on methods which fit into the framework of a patient-oriented approach to medicine. Such methods include clinical clerkships, around which all clinical teaching in undergraduate medical school is developed, and the newer method of preceptorships in which students work directly with physicians in general practice. Also, the assignment of students as family medical advisers will enable them to see patients in their natural habitat and to gain first-hand knowledge about the chronic illnesses and the social aspects of illness.

\section{The Future Physician}

Consideration of the patient-oriented approach to medicine raises questions which prompt a reevaluation of the physician's role in society and of the kind of person the physician might ideally be. In addition to his specialized responsibilities, the physician has an opportunity to function as a leader and should be sensitive to the issues and problems of his community and to the people in it. The medical school recognizing this is paying increasing attention to the development of the medical stu. 
dent both as a mature individual and as a wellqualified member of the medical profession.

In any list of the assets which the future physician should bring with him to medical school, the factors indicating success in interpersonal relationships are of extreme importance. Some critics question whether current admissions policies result in the selection of students who are potentially most capable of fulfilling the responsibilities of socially oriented physicians as well as of scientists.

The conference, in addition to pointing out the loss of good students through restrictive admissions policies and artificial quota systems, strongly urged the need for a shift in emphasis in selection criteria. The current emphasis on scholastic grades places too great a premium on preparation in the basic sciences, on interest in things rather than in people, without sufficient regard for the personal and social aspects of medicine.

It was suggested that a psychiatrist be included on the admissions committee of a medical school to help in the interviewing and the selection of students. It was also suggested that the medical school faculty confer frequently with the advisers of premedical school students to interpret to them the need of the future physician for broader areas of knowledge and understanding.

The emphasis on the medical student as a well-rounded individual with a liberal college education, an individual aware of his responsibilities to society and sensitive to the pressures and problems of his fellow citizens, carried over into the conference discussions about the general nature of medical education. Personality growth, stimulation of thought and action on the part of students, and close faculty-student relationships were stressed as primary goals of medical education.

\section{Creative Teaching}

The trend away from didactic instruction and toward freedom for the student to express doubt and criticism is further complicated by the increasing difficulty of imparting the full fund of current medical knowledge in a 4-year course of study. The almost overwhelming amount of knowledge the medical student must now acquire intensifies the natural anxiety he experiences as he begins to realize the responsibilities he is assuming as a future physician. The aim of medical training, therefore, must be to develop insight into the principles, and skill in the application, of working methods.

Medical school teaching provides the opportunity for a creative kind of teaching. In building the medical faculty, more consideration should be given to teaching ability as well as to research and clinical interests. To provide the student with a milieu in which he can develop his interests, acquire knowledge in a coordinated fashion, and at the same time gain experience in dealing with human beings, the individual faculty members, the various departments, and the administration of the medical school must pool their resources-philosophical, scientific, and professional-as well as those of personnel, equipment, and space.

\section{Solution}

In conclusion, the conference report emphasized that "the techniques of teamwork and group action extend beyond cooperative work with physicians. Many of the problems cited ... cannot be solved by caring for the individual patient or family but must be approached on a community-wide basis. The physician's work must more and more be integrated with the broad program of preventive medicine and maintenance of health. This is so because the skill and knowledge required for the mastery of major problems of 'social engineering' exceed the resources of the individual physician. Knowing his limitations, he must be able to assess the resources of other groups and to join in teamwork for community betterment, contributing his special skills and knowledge about stress and illness, and recognizing the contributions of other disciplines."

In the light of what psychiatry has already learned about how people function and how psychic, somatic, and social factors are inextricably interrelated, the integrative force of psychiatry and medical education should be a force for better mental and physical health.

\section{-Seymour D. Vestermark, M.D.}

